\title{
Second Response to Samuel Wheeler
}

Paul Livingston

University of New Mexico

I wish to thank Prof. Wheeler again for his extremely helpful discussion of these issues of language, meaning, and interpretation. In this final response, I can be relatively brief, for I believe we are actually in substantial agreement about the contours of Davidson's project and (for the most part, at least), about their implications for the possibility of a philosophical theory of linguistic meaning.

Professor Wheeler notes correctly that Quine and Davidson's results imply that all items relevant to interpretation - including thoughts and intentions - are "languagelike." That is, there are no "interpretation-enders," no items capable of accounting for the meaning of words or sentences that are not themselves open to interpretation and indeterminacy. This implies, as well, that there can be no theory of meaning that explains it "from outside content" - that is, there is no theory that explains the meaning of sentences and terms determinately in terms of non-semantic or non-"language-like" facts or items. This is McDowell's point against Dummett. Dummett's claim that a theory of meaning should be 'immodest' - capable of conferring a grasp of the concepts whose truth-conditional semantics it expresses - is just the requirement that a theory of meaning characterize the content of the sentences it treats in a way that would make this content intelligible from neutral materials "outside content." McDowell argues, quite rightly on my view, that Davidsonian theories of meaning cannot be construed as capable of doing this. Because of indeterminacy, there is no theoretical route from "outside content" - from bare facts or phenomena, whether of a physical, neurophysiological, or behavioral kind - to the meaning of ordinary expressions.

In this sense, the consequences of indeterminacy are quite profound: as a result of indeterminacy, it is impossible to theorize semantic content in neutral terms, and thus impossible to theorize meaningful language in the way we can normally theorize (other) natural phenomena. On the other hand, as Wheeler also rightly points out, this inability to theorize meaning does not have dire consequences for our ability to understand each other on an everyday basis. That is, it remains quite right to say, for instance, that we 
can correctly attribute to someone possession of the concept "square" if they exhibit the capacity to recognize squares, and that the rule for use of the term "square" is: Call something "square" if and only if it is a square. Indeterminacy does not threaten to raise major problems, in practice, for the success of attributions of this kind. ${ }^{1}$ The problem that McDowell raises is just that (as Wheeler notes) in making these attributions, we (necessarily) presuppose our own ability to use the concepts whose grasp we are attributing. On Davidson's account, the necessity that we make this presupposition is, in large part, the necessity of applying the "principle of charity." Nevertheless, as Wheeler also recognizes, we do not thereby gain an understanding, explanation or account of what is involved in our use of language in terms purified of that presupposition. ${ }^{2}$

Does the theoretical problem that McDowell raises, then, "get a purchase" on Davidson's account of concept-attribution? It does not, in the sense that it does not suggest any deep or general practical problem for our ability to attribute concepts and understand one another. However, I believe it does, in the sense that it demonstrates the inherent indeterminacy involved in any theoretical attempt to characterize the use of language in factual or descriptive terms that do not largely presuppose that very use. It is, doubtless, true that the presupposition of shared use, as well as a shared network of practices and attributions of rationality and agency, "goes very far back indeed" (Wheeler 4). Without it, we could not even begin to make sense of each other. But to say that this network of practices and attributions is vast and pervasive in human life is not to say that we have, or can have, a theoretical explanation or account of it. Moreover, at those ordinary junctures of human life where the meaning of our existing practices or their application in the future is a subject of explicit discussion, our inability (necessary though it may be, on Quine and Davidson's telling) to theorize meaning in neutral terms, or understand meaning as part of the natural world in the sense in which physical matter and forces are part of the natural world, may certainly become a cause for (ordinary) concern.

In the last paragraph of his response, Wheeler seems to recognize that the question of how we gain mastery of language is an important one, even if the results of Quine and Davidson tend to suggest that we cannot give a philosophical answer to it. 
He implies that where philosophical theory fails, empirical theories, for instance drawn from developmental psychology and linguistics, may succeed. Without delving deeply into this point, my guess is that the problem for the attempt to theorize meaning that Quine and Davidson point out cannot easily be solved by any empirical theory, unless such a theory presupposes, to a large degree, what is to be explained. The point of indeterminacy, after all, is that the provision of any number of (uninterpreted) empirical facts still leaves room for a variety of interpretations; but if we begin with facts already interpreted semantically (for instance as facts manifesting the presence of a "disposition to acquire meanings" or to "attribute intentional states") we presuppose what is to be explained

\footnotetext{
${ }^{1}$ There may still be residual and relatively minor indeterminacies, as, for instance, when someone, against the backdrop of a set of beliefs and capacities that we take to be largely similar with our own, utters a sentence which may either be asserting (what we take to be) a falsehood or using words in a non-standard way. That this limited kind of indeterminacy is all that remains, in practice, if we may assume (as we ordinarily do) a background of shared practice and belief, is all that I meant in the passage that Wheeler quotes on page I of his document.

${ }^{2}$ Against the shared backdrop of ordinary practices and attributions of belief, it is correct to say that we can attribute the concept "square" to someone who normally, and without glaring counterexamples, manifests the ability to recognize squares and to use the term "square" to designate them. However, this is not the same as to say that such a person has a term (namely "square") "whose extension is all and only the squares." For (given finite time and resources) we are not in a position to determine the relationship of a speaker's past and present observed usage to an infinite totality such as the set of all squares. This is a way of putting the force of the "paradox" that Wittgenstein mentions at PI 20I; the resolution is, in part, that we may legitimately attribute mastery of the concept "square," against the backdrop of a constitutive (charity) assumption of shared practices and largely shared beliefs, even if we admittedly have no way of determining ("all at once") the totality of its extension.
} 